Artículo de revisión

\title{
Riesgo biomecánico por sobrecarga estática y presencia de trastornos musculoesqueléticos en odontólogos durante su práctica clínica asistencial. Una revisión narrativa
}

\section{Ergonomic risk by static overload and musculoskeletal disorders in dental practice: a review}

\section{Risco biomecânico devido a sobrecarga estática e presença de distúrbios musculosqueléticos em dentistas durante sua prática clínica de assistência. Uma revisão narrativa}

\author{
Vicenzo Vicente Agredo-Silva ${ }^{1 凶}$, María Camila Arias-Arango ${ }^{凶}$, Juliana Villegas- Monsalve ${ }^{2}$, \\ Natalia Zapata-Ortega ${ }^{3 凶}$, Ricardo Sergio Zapata-Martínez ${ }^{3 凶}$, Mariana Zuluaga-Tamayo ${ }^{3 凶}$ \\ ${ }^{1}$ Fisioterapeuta. MSc Salud Ocupacional, MSc Administración, Esp Docencia Universitaria e Investigación. Estudiante de segundo año \\ de doctorado en Salud Publica, Docente. Universidad CES. \\ ${ }^{2}$ Estudiantes noveno semestre. Facultad de Fisioterapia. Universidad CES. \\ ${ }^{3}$ Fisioterapeutas. Universidad CES.
}

Fecha correspondencia:

Recibido: noviembre de 2020

Aprobado: septiembre de 2021

Forma de citar:

Agredo-Silva VV, Arias-Arango MC, Villegas- Monsalve J, ZapataOrtega N, Zapata-Martínez RS, Zuluaga-Tamayo M. Riesgo biomecánico por sobrecarga estática y presencia de trastornos musculoesqueléticos en odontólogos durante su práctica clínica asistencial. Una revisión narrativa. Rev. CES Odont 2021; 34(2): 123-138.

https://doi.org/10.21615/ cesodon.5989

Open access

\section{Resumen}

Los trastornos musculoesqueléticos son lesiones que afectan el movimiento corporal, incluyendo músculos, tendones, ligamentos, entre otros. Estos trastornos son unos de los problemas más importantes en la práctica clínica odontológica, ocasionados en su mayoría por sobrecarga física tanto dinámica como estática, en particular para esta última, toma relevancia la postura mantenida en posición bípeda o sedente durante la realización de procedimientos extremadamente precisos en un espacio de trabajo tan pequeño como lo es la cavidad oral; por esto, se pretende con base en la literatura existente, describir el riesgo biomecánico por sobrecarga estática y los trastornos musculoesqueléticos en odontólogos. Con esta intención se realizó una revisión narrativa de la literatura científica al respecto en español e inglés de productos de investigación de los últimos diez años, utilizando descriptores DeCS y $\mathrm{MeSH}$, en fuentes 
(C) Derecho de autor

Licencia creative commons

Ética de publicaciones

Revisión por pares

Gestión por Open Journal

System

DOI: $10.21615 /$ cesodon.5989

ISSNe 2215-9185

ISSN 0120-971X

Publica con nosotros académicas y científicas de Pubmed, Medline, Scopus y Google académico en donde se seleccionaron 50 artículos teniendo como resultado principal que si existe una relación entre riesgo biomecánico y sobrecarga estática con la aparición de trastornos musculoesqueléticos.

Palabras Clave: Riesgo biomecánico; riesgo ergonómico; ergonomía; trastornos musculoesqueléticos; odontólogos.

\section{Abstract}

Musculoskeletal disorders are injuries that affect body movement, including muscles, tendons, ligaments, among others. These disorders are one of the most important problems in dental clinical practice, caused mostly by static overload due to prolonged posture in a standing or sitting position when performing extremely precise procedures in a workspace as small as the oral cavity; For this reason, based on the existing literature, it is intended to describe the biomechanical risk due to static overload and musculoskeletal disorders in dentists. With this intention, a narrative review of the scientific literature on the matter in Spanish and English of research products of the last ten years was carried out using DeCS and MeSH descriptors, in academic and scientific sources of PubMed, Medline, Scopus and academic Google in where 50 articles were selected, the main result being that there is a relationship between biomechanics factors and static overload with the use appearance of musculoskeletal disorders.

Keywords: biomechanical risk; ergonomics; musculoskeletal disorders; dentist; ergonomic risk.

\section{Resumo}

As desordens musculoesqueléticas são lesões que afetam os movimentos do corpo, incluindo músculos, tendões, ligamentos, entre outros. Esses distúrbios constituem um dos problemas mais importantes na prática clínica odontológica, ocasionados principalmente pela sobrecarga física, tanto dinâmica quanto estática, em particular para esta última, torna-se relevante a postura mantida na posição bípede ou sentada durante procedimentos extremamente precisos. tão pequeno quanto a cavidade oral; Portanto, pretende-se, com base na literatura existente, descrever o risco biomecânico devido à sobrecarga estática e distúrbios musculoesqueléticos em dentistas. Com esse intuito, foi realizada uma revisão narrativa da produção científica sobre o assunto, em espanhol e inglês, de produtos de pesquisa dos últimos 
dez anos, utilizando os descritores DeCS e MeSH, em fontes acadêmicas e científicas do Pubmed, Medline, Scopus e Google acadêmico onde foram selecionados 50 artigos, sendo que o principal resultado é que existe uma relação entre o risco biomecânico e a sobrecarga estática com o aparecimento de distúrbios musculoesqueléticos.

Palavras-chave: risco biomecánico; risco ergonómico; ergonomía; distúrbios musculoesqueléticos; dentistas.

\section{Introducción}

Según la Tabla de Clasificación de Actividades Económicas para el Sistema General de Riesgos Profesionales descrita en el decreto 1607 de 2002, la práctica odontológica es considerada una actividad económica de bajo riesgo ocupacional, nivel 1 sobre 5, para riesgos laborales en Colombia ${ }^{(1)}$; sin embargo, una variedad de factores contribuye a la presencia de trastornos musculoesqueléticos (TME) entre los odontólogos, incluidos los factores de riesgo biomecánico, los cuales son un conjunto de atributos o elementos que aumentan la posibilidad de que los odontólogos expuestos desarrollen una lesión ${ }^{(2,3)}$.

Dentro de los factores de riesgo biomecánico podemos encontrar los relacionados con las posturas, tales como: mantenidas, cuando se adopta una postura biomecánicamente correcta por 2 horas o más o biomecánicamente incorrecta cuando se mantienen por 20 minutos o más; forzadas, la cual se considera cuando se sobrepasa el 50\% del rango articular; prolongadas, cuando se adopta la misma postura por el $75 \%$ o más de la jornada laboral y anti gravitacionales donde se posiciona un segmento del cuerpo en contra de la gravedad ${ }^{(4)}$.

Teniendo en cuenta la actividad práctica del odontólogo se decide enfatizar en la sobrecarga estática, la cual está determinada por las posturas y se relaciona con las contracciones musculares mantenidas, otra causa es la sobrecarga dinámica, la cual está determinada por el esfuerzo muscular, los desplazamientos, el manejo de cargas y los movimientos repetitivos (4), sin embargo, en esta revisión no profundizaremos en este tipo de carga física.

Mantener una posición estática mientras se realizan procedimientos extremadamente precisos en un espacio de trabajo pequeño, durante largos períodos de tiempo son algunas de las causas de los TME ${ }^{(5)}$. La prevalencia de los TME en el personal dental sugiere que las regiones más prevalentes incluyen el cuello $(58,5 \%)$, espalda baja $(56,4 \%)$, hombro $(43,1 \%)$ y parte superior de la espalda $(41,1 \%)$, además se pueden presentar en menor porcentaje en muñecas y manos ${ }^{(6-8)}$. 
Los TME son lesiones que afectan el movimiento corporal humano, esto incluye, músculos, tendones, ligamentos, nervios, discos y vasos sanguíneos, ocasionados en su mayoría por sobrecarga estática relacionadas con la adopción de posturas mantenidas y/o forzadas tanto en posición bípedo como en sedente en la práctica odontológica rutinaria ${ }^{(9)}$.

Este artículo de revisión pretende narrar, con base en la literatura existente, la relación encontrada entre el riesgo biomecánico por sobrecarga estática y la presencia de trastornos musculoesqueléticos en odontólogos durante su práctica clínica asistencial.

\section{Materiales y métodos}

La búsqueda de la literatura incluyó investigaciones originales, artículos de revisión y revisiones sistemáticas en los idiomas inglés y español en revistas indexadas incluidas en las bases de datos de Scopus, Pubmed, Medline, y Google Académico publicados entre los años 2010 y 2020. Las palabras claves incluidas fueron riesgo biomecánico, riesgo ergonómico, ergonomía, trastornos musculoesqueléticos y odontólogos, utilizando para ello los indicadores AND. Se excluyeron artículos repetidos y artículos que no se encontraron disponibles en texto completo.

En el gráfico 1 se ilustra el número de artículos incluidos. Del total de 192 estudios encontrados con la estrategia de búsqueda descrita, fueron seleccionados 57 para lectura completa de los cuales 50 forman parte de esta revisión, debido a su calidad metodológica y a la relevancia de sus resultados. 


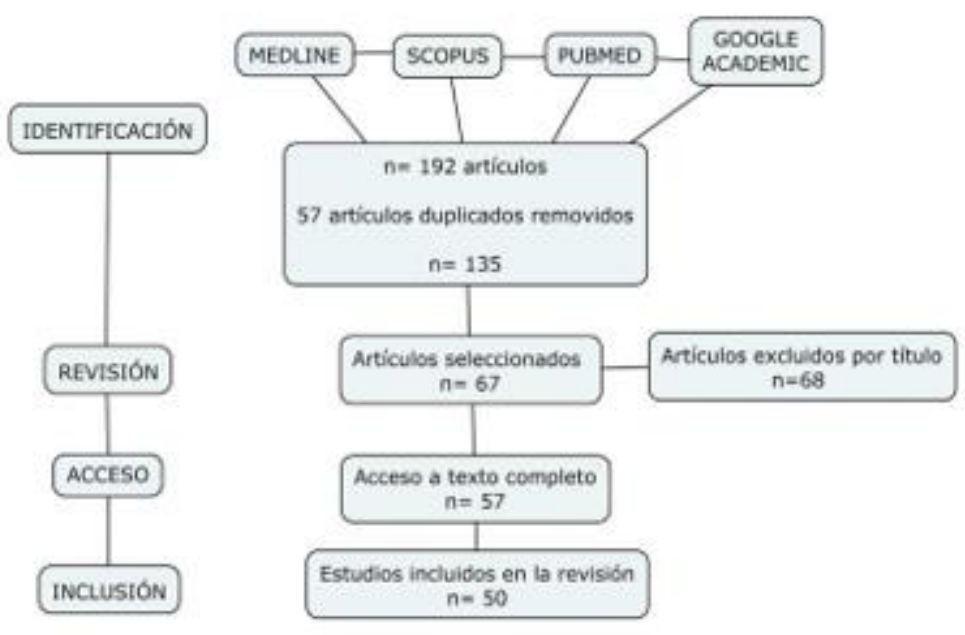

Gráfico 1. Flujograma de revisión y selección.

Fuente: elaboración propia utilizando CmapTools.

\section{Trastornos Musculoesqueléticos}

Los TME están caracterizados por disconfort, hormigueo, entumecimiento, hinchazón, movimiento limitado o dolor en articulaciones, músculos, tendones y otros tejidos blandos ${ }^{(5,7,10)}$. Otros síntomas pueden manifestarse como disminución de la función muscular, alteración de la conducción nerviosa, distensiones, roturas de músculos, tendones y ligamentos, y microfractura de los huesos. Algunos TME específicos se han identificado como síndrome del túnel carpiano, tendinitis y tenosinovitis de Quervain ${ }^{(11-13)}$.

La práctica odontológica implica actividades precisas y controladas que requieren especial atención, concentración y paciencia del odontólogo ${ }^{(14,15)}$. Es fácil inferir que debido a que los odontólogos deben desempeñar sus labores en una parte del cuerpo tan específica y pequeña como la cavidad oral hace que adopten posturas mantenidas que pueden causar molestias musculoesqueléticas paulatinas ${ }^{(16,17)}$.

El cincuenta por ciento de los músculos del cuerpo se contraen para mantener estas posturas estáticas mientras resisten la gravedad ${ }^{(18,19)}$. Diferentes estudios indican que más del $90 \%$ de los odontólogos han sufrido alguna vez de malestar o dolor en al menos una región del cuerpo $(10,17)$, especialmente los dolores de espalda baja (región lumbar), espalda alta 
(región torácica) y cuello (región cervical), que son frecuentes y fuertemente asociados con su práctica clínica ${ }^{(20,21)}$.

Las posturas incómodas identificadas con más frecuencia entre los profesionales de la odontología son: flexión del cuello; inclinación y rotación del tronco; elevación de hombros; aumento de las curvaturas de la columna vertebral y posicionamiento incorrecto de las extremidades inferiores ${ }^{(17,20-22)}$. Incluso muchos de estos trastornos pueden empezar su aparición desde la etapa estudiantil sin variar de manera significativa los resultados respecto a la etapa profesional ${ }^{(23,24)}$.

Los estudios han demostrado que los odontólogos tienen una alta frecuencia de TME desde hace décadas ${ }^{(25)}$. Una revisión reciente de la literatura examinó la prevalencia y los factores de riesgo de TME en odontólogos y sugirió que la prevalencia del dolor musculoesquelético general entre los profesionales dentales varía entre $64 \%$ y $93 \%{ }^{(26,27)}$.

Los TME se consideran de origen multifactorial ${ }^{(28)}$. Posturas estáticas prolongadas e inadecuadas, repetitividad, contracciones mantenidas, iluminación deficiente, posicionamiento inapropiado tanto del paciente como del profesional dental, falta de asistente dental, las demandas visuales para abarcar toda la cavidad oral y sus componentes y el estrés conducen y/o incrementan de manera significativa el desarrollo de TME ${ }^{(7,29-31)}$. Las mujeres muestran una mayor frecuencia de dolor en la zona cervical, lumbar, dorsal y en las muñecas, exponiéndolas a un mayor riesgo de lesión ${ }^{(32,33)}$.

Otros factores de riesgo que aumentan significativamente estas probabilidades son la edad, la duración de la cita, los años de experiencia laboral y la especialidad. En este sentido los periodoncistas son más propensos a informar TME (dolor en hombros) relacionados con el trabajo, seguidos por los endodoncistas. En contraste, los cirujanos maxilofaciales y ortodoncistas tienen menores probabilidades de desarrollar TME de este tipo en comparación con los odontólogos generales ${ }^{(34)}$. 


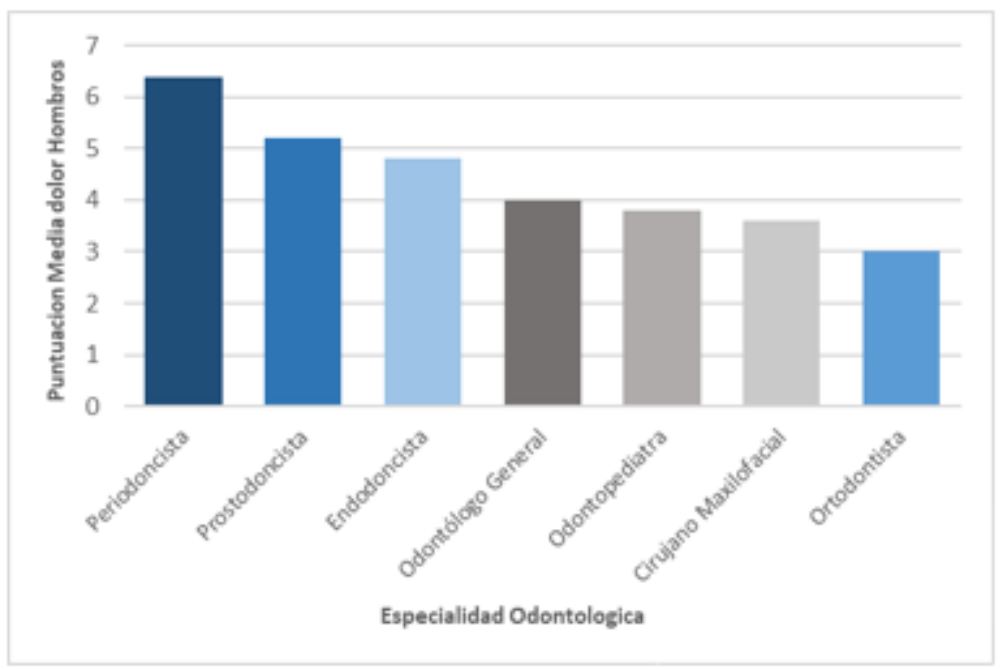

Gráfico 2. Media dolor de hombros según especialidad.

Fuente: elaboración propia utilizando los datos y referencias de Meisha DE, Alsharqawi NS, Samarah AA, Al-Ghamdi MY (34).

\section{Factores ergonómicos y su asociación con los TME en odontólogos}

Para una práctica odontológica óptima debe haber un equipo y un entorno ergonómico ${ }^{(14)}$. La ergonomía es una ciencia que se ocupa de cómo adaptar el carácter anatómico, fisiológico y psicológico de un trabajo de tal manera que mejore la eficiencia y el bienestar humano ${ }^{(20)}$. Esta disciplina permite además el control de accidentes y enfermedades laborales mediante la reducción de riesgos ${ }^{(35)}$.

Se ha prestado una atención insignificante al entorno dental, incluido el diseño espacial, el espacio libre y el alcance de herramientas y equipos, las ubicaciones de almacenamiento, el acceso, el movimiento del usuario y el flujo de trabajo ${ }^{(36)}$. Una de las principales causas para desarrollar TME es la inconsciencia para adoptar posturas seguras durante las tareas laborales ${ }^{(17)}$. En este sentido para el campo de la ergonomía aplicada en odontología la postura de trabajo ideal resulta ser uno de los temas más discutidos ${ }^{(14)}$.

Estas condiciones del entorno y la forma de trabajo de los odontólogos que pueden constituirse en factores de riesgo para la salud y la seguridad de los profesionales, logran ser analizadas en el marco de diferentes tipos de ergonomía. Así la ergonomía física tiene en cuenta los aspectos relacionados con las posturas adoptadas, el posicionamiento la silla y la de los pacientes, así como el manejo de los instrumentos de trabajo, entre otros. La ergonomía cognitiva identifica 
factores derivados de la carga mental o la falta de habilidades eficientes; mientras que la ergonomía organizacional da cuenta de los factores que tienen relación con la estructura, las políticas, los procesos laborales y administrativos asociados con horarios, intervenciones estipuladas por hora o por día, entre otros ${ }^{(11,37)}$.

En este sentido, diferentes estudios han demostrado que los odontólogos reportan problemas de salud más frecuentes y peores que otros profesionales médicos. Así, las posiciones incómodas asumidas durante el trabajo odontológico tienen efectos nocivos en el sistema musculoesquelético. Por ejemplo, las posiciones estáticas mantenidas en bípedo o sedente con rotación espinal pueden conducir a un aumento en la presión del disco intervertebral y originar espasmos musculares ${ }^{(38)}$.

La postura ideal de un odontólogo le confiere, por un lado, unas condiciones de trabajo óptimas como lo es un adecuado acceso, visibilidad y control en la boca y, por otro lado, comodidad física y psicológica durante la ejecución de los actos clínicos. Proporcionando más energía de trabajo, un menor nivel de estrés, mayor confort, ausencia de dolor y tensión muscular y un menor riesgo de errores terapéuticos. Una postura incorrecta induce fatiga prematura, dolor, estrés y una actitud negativa para trabajar, un alto riesgo de TME y mala calidad del trabajo ${ }^{(14)}$.

La actividad profesional en odontología es influenciada por la ergonomía, ya que es dependiente de factores físicos y mecánicos como fuerza, precisión y vibración. Una serie de características de los instrumentos dentales, como la forma, diámetro, tamaño, peso, textura del mango, herramientas dinámicas o estáticas también pueden representar factores importantes que influyen en TME relacionados con el trabajo en odontología ${ }^{(39,40)}$.

En general, uno de los principales problemas que no permiten la adopción de posturas ergonómicas entre los odontólogos es la formación insuficiente en ergonomía, las unidades dentales que funcionan de manera inadecuada y la falta de personal auxiliar ${ }^{(41)}$. La información ergonómica suficiente y la capacitación en prevención no están adecuadamente integradas en los programas de formación dental; los estudios apoyan la idea de que la intervención ergonómica durante la educación es necesaria antes de que los estudiantes se embarquen en sus carreras profesionales ${ }^{(42)}$.

Cuando las posturas habituales se desarrollan a partir del trabajo en entornos clínicos desafiantes, se vuelve más difícil modificar esos hábitos con el tiempo. Sin embargo, el uso periódico de la toma de fotografías de las posturas de trabajo para autoevaluaciones de 
ergonomía puede ayudar a mitigar esas posturas ${ }^{(43)}$. Es importante considerar también el uso de nuevas herramientas para el mejoramiento de las posturas durante el trabajo, el uso de dispositivos ópticos para facilitar la agudeza visual, agarres ergonómicos para herramientas dentales para reducir los dolores en mano y muñeca; y cambio de posición del paciente para permitir que los odontólogos adopten una postura neutral en el trabajo ${ }^{(36)}$.

La prevención y el tratamiento relacionados con la odontología y el dolor musculoesquelético se enfocan en adoptar posturas adecuadas para sentarse y realizar estiramientos y pausas periódicas ${ }^{(44)}$. Además, se ha demostrado en estudios previos que el uso de lupas mejora la postura, y también se observó un efecto protector para aquellos que informaron haber recibido educación ergonómica con respecto a la posición del operador y la silla del paciente ${ }^{(12)}$.

En un estudio se menciona que la ubicación del dolor entre los encuestados parecía estar relacionado con el tipo de sillas que utilizaban. Los encuestados que usaban silla giratoria tenían mayor prevalencia de trastornos musculoesqueléticos relacionados con el trabajo, un $54 \%$ de ellos refirió dolor en la región del cuello, y un $61 \%$ en la espalda baja ${ }^{(45)}$. Generalmente, estos problemas se encuentran relacionados con un mal diseño del puesto de trabajo ${ }^{(46)}$.

Otro estudio proporcionó algunos puntos para trabajar con una buena postura, que incluye el uso de silla ajustable con soporte lumbar, torácico y de antebrazos, mantenimiento de una postura erguida, colocación de los pies apoyados en el piso con la silla cerca del paciente y posiciones de trabajo alternas entre sedente y bípedo; los encuestados que siguieron estas recomendaciones refirieron disminución del dolor y de incomodidad musculoesquelética (47).

A pesar de los numerosos avances técnicos de los últimos años, existe una creciente evidencia de que los profesionales de la odontología todavía están predispuestos a una serie de riesgos laborales. Estos incluyen exposición a infecciones, lesiones oculares, vibraciones, exposición a radiaciones, materiales dentales, ruido, afecciones psicológicas y TME ${ }^{(48)}$.

A pesar de las mejoras en la ergonomía, como el lugar de trabajo y el diseño de herramientas, los TME relacionados con el trabajo todavía representan un importante problema de salud ocupacional en la odontología moderna $(49,50,30)$. Estas lesiones podrían comenzar a aparecer al inicio de su práctica clínica y acompañarlos por el resto de su vida profesional, adquiriendo un estilo de vida poco saludable en su entorno laboral ${ }^{(35)}$. 


\section{Discusión}

Diferentes estudios dan por sentado que las características sociodemográficas como la edad, género, peso, altura, entre otros, tienen poca o ninguna relevancia o influencia estadísticamente significativa relacionado con los TME ${ }^{(17,50)}$. En contraste, en los últimos años se ha dado una mayor importancia a la relación que tienen dichas características con el desarrollo de los TME y que si pueden impactar de manera significativa, aunque esta situación no tendría tanta relevancia si no se acompañan por los demás factores de riesgo físico presentes en la odontología, por lo tanto, el origen de los TME es multifactorial y biopsicosocial.

Un aspecto controversial con lo que previamente considerábamos es que dos de los estudios revisados mencionan que la aparición de sintomatología se puede dar desde la etapa estudiantil, sin embargo, consideramos que el tiempo de exposición (factor al que le dan sobrevalor en los estudios) no es suficiente comparado con los de la etapa profesional para generar los TME.

Aunque un gran porcentaje de odontólogos presentan algún tipo de TME, los cuales son responsables del ausentismo laboral, la mala salud y la jubilación anticipada ${ }^{(36,51)}$; muy pocos recurren a la ayuda de otros profesionales de la salud como médicos, fisioterapeutas, entre otros ${ }^{(29)}$, esto puede suceder al no querer disminuir el tiempo de exposición representado en pacientes atendidos por día, ya que puede implicar disminución en los ingresos económicos.

En este sentido, un número estadísticamente significativo de odontólogos que habían experimentado molestias musculoesqueléticas de intensidad moderada o mayor, utilizaron el ejercicio como terapia o se automedicaron con más frecuencia que aquellos con dificultades leves o nulas ${ }^{(48,52)}$ teniendo en cuenta que no se toman medidas correctivas de manera precoz, incurriendo en el constante trauma acumulativo que se agrava con el tiempo volviéndose de difícil manejo; es ahí que se tornan imprescindibles las ayudas tomadas, ya sean autoimpuestas o por parte de otro profesional. Los TME son una causa común de discapacidad relacionada con el trabajo, con consecuencias financieras sustanciales debido a la compensación de los trabajadores y los gastos médicos ${ }^{(38)}$. Debido a la alta prevalencia y su impacto en la vida, la salud y la productividad, TME representan una condición de salud negativa importante ${ }^{(53)}$.

Las limitaciones de la mayoría de la literatura revisada se centran en el tamaño de la muestra, la especialidad odontológica estudiada, el tipo de instrumentos utilizados por los profesionales, la posición adoptada al trabajar y el tiempo de exposición, por lo cual los resultados no pueden ser generalizados o extrapolados a toda la población de odontólogos. 


\section{Conclusiones}

Es evidente la existencia de la relación entre los factores de riesgo biomecánicos y ergonómicos con la presencia de TME, sin embargo, aunque las tareas laborales realizadas por los odontólogos son similares, la forma de realizarlas, el conocimiento previo de ergonomía, las medidas tomadas para la prevención y promoción pueden ser diferentes en cada población y en cada especialidad odontológica.

Adoptar posturas adecuadas en la práctica clínica y tener un ambiente de trabajo favorable podría reducir la frecuencia de lesiones del sistema musculoesquelético evitando una jubilación anticipada de la profesión; por esto es de vital importancia promover programas de formación y prevención en salud ocupacional en cuanto a posturas ergonómicas que deben adquirir los profesionales en odontología durante sus prácticas clínicas, originando así estilos de vida saludables.

Según artículos revisados, se menciona que las mejoras ergonómicas en el lugar de trabajo, por ejemplo, ajustes de la silla y ubicación de esta con respecto al paciente, correcciones posturales, practicar ejercicios físicos y la reducción del estrés ocupacional son efectivos y necesarios en la reducción de los TME entre los odontólogos, además es preciso consultar a un profesional a tiempo para evitar a futuro el ausentismo laboral.

\section{Agradecimientos}

Agradecemos a la biblioteca Fundadores de la Universidad CES por las facilidades en el acceso a las bases de datos y revistas indexadas, que nos permitió la búsqueda y lectura de los diferentes artículos científicos y material bibliográfico que sirvieron de base para la construcción del presente artículo.

\section{Referencias}

1. Ministerio de Trabajo y Seguridad Social. Decreto 1607 de 2002. Tabla de Clasificación de Actividades Económicas para el Sistema General de Riesgos Profesionales. Función Pública. [Internet]. [citado 7 de octubre de 2020].

2. Al-Rawi NH, Yousef H, Khamis M, Belkadi O, Ahmed S, Ali S. Vertebral malalignment among male dentists with workrelated musculoskeletal pain in the United Arab Emirates. J Contemp Dent Pract. 2018;19(7):773-7. 
3. Ministerio De La Protección Social. Guía de Atención Integral Basada en la Evidencia para Desórdenes Músculo esqueléticos (DME) relacionados con Movimientos Repetitivos de Miembros Superiores (Síndrome de Túnel Carpiano, Epicondilitis y Enfermedad de De Quervain) GATI-DME. 2006. Bogotá, D.C. 2006.

4. Consejo superior de la judicatura. SIGCMA. Programa de gestión para la intervención de riesgo biomecánico relacionado con desórdenes músculo esqueléticos (DME). 2019.

5. Aljanakh M, Shaikh S, Siddiqui AA, Al-Mansour M, Hassan SS. Prevalence of musculoskeletal disorders among dentists in the Ha'il Region of Saudi Arabia. Ann Saudi Med. 2015;35(6):456-61.

6. Lietz J, Ulusoy N, Nienhaus A. Prevention of musculoskeletal diseases and pain among dental professionals through ergonomic interventions: A systematic literature review. Int J Environ Res Public Health [Internet]. 2020;17(10).

7. Roshene R, Loganathan S. Ergonomics and musculoskeletal disorder as an occupational hazard in dentistry- a pilot study. J Pharm Sci Res. 2017;9(5):712-5.

8. Pradeep-Kumar R, Saraswathi S, Thangavelu L. Prevalence of musculoskeletal disorder and experience among dental practitioners in Chennai - A cross-sectional survey. Drug Invent Today. 2019;12(1):69-73.

9. Harris ML, Sentner SM, Doucette HJ, Brillant MGS. Musculoskeletal disorders among dental hygienists in Canada. Can J Dent Hyg. 2020;54(2):61-7.

10. Humann P, Rowe DJ. Relationship of Musculoskeletal Disorder Pain to Patterns of Clinical Care in California Dental Hygienists. J Dent Hyg JDH. 2015;89(5):305-12.

11. Mulimani P, Hoe VCW, Hayes MJ, Idiculla JJ, Abas ABL, Karanth L. Ergonomic interventions for preventing musculoskeletal disorders in dental care practitioners. Cochrane Database Syst Rev [Internet]. 2018;2018(10).

12. Hayes MJ, Taylor JA, Smith DR. Predictors of work-related musculoskeletal disorders among dental hygienists. Int J Dent Hyg. 2012;10(4):265-9.

13. Ohlendorf D, Maltry L, Hänel J, Betz W, Erbe C, Maurer-Grubinger C, et al. SOPEZ: Study for the optimization of ergonomics in the dental practice - Musculoskeletal disorders in dentists and dental assistants: A study protocol. J Occup Med Toxicol [Internet]. 2020;15(1). 
14. Pîrvu C, Pătraşcu I, Pîrvu D, Ionescu C. The dentist's operating posture - ergonomic aspects. J Med Life. 2014;7(2):177-82.

15. Bennadi D, Reddy V, Thummala NR. Preventive and curative measures adopted by dentists to combat occupational hazards-a cross sectional study. Int J Pharm Pharm Sci. 2015;7(10):416-8.

16. Kang HK. Research on the level of job stress and musculoskeletal disorders and the presence of pain from work in dental hygienists. Int J Bio-Sci Bio- Technol. 2015;7(5):283-96.

17. Batham C, Yasobant S. A risk assessment study on work-related musculoskeletal disorders among dentists in Bhopal, India. Indian J Dent Res. 2016;27(3):236-41.

18. Gupta D, Devaki M, Dommaraju N, Srinivas KT, Patil AA, Momin RK, et al. Musculoskeletal pain management among dentists: An alternative approach. Holist Nurs Pract. 2015;29(6):385-90.

19. Sakzewski L, Naser-Ud-Din S. Work-related musculoskeletal disorders in dentists and orthodontists: A review of the literature. Work. 2014;48(1):37-45.

20. Gupta S. Ergonomic applications to dental practice. Indian J Dent Res. 2011;22(6): 816-22.

21. Tirgar A, Javanshir K, Talebian A, Amini F, Parhiz A. Musculoskeletal disorders among a group of Iranian general dental practitioners. J Back Musculoskelet Rehabil. 2015;28(4):755-9.

22. Fernandez de Grado G, Denni J, Musset A-M, Offner D. Back pain prevalence, intensity and associated factors in French dentists: a national study among 1004 professionals. Eur Spine J. 2019;28(11):2510-6.

23. Dos Santos RR, Garbin CAS, Batista JA, Saliba TA, Garbin AJI. Prevalence of musculoskeletal pain in dental students and associated factors. Braz J Oral Sci. 2019; 18:1-11.

24. Garcia PP, Presoto CD, Maroco J, Campos JA. Work-related activities that may contribute to musculoskeletal symptoms among dental students: validation study. Med Lav. 2016;107(3):235-42. 
25. Barry RM, Spolarich AE, Weber M, Krause D, Woodall WD, Bailey JH. Impact of Operator Positioning on Musculoskeletal Disorders and Work Habits Among Mississippi Dental Hygienists. J Dent Hyg JDH. 2017;91(6):6-14.

26. Gupta D, Bhaskar DJ, Gupta KR, Karim B, Kanwar A, Jain A, et al. Use of complementary and alternative medicine for work related musculoskeletal disorders associated with job contentment in dental professionals: Indian outlook. Ethiop J Health Sci. 2014;24(2):117-24.

27. Mikuláková W, Kendrová L, Homzová P, Urbanová K, Labunová E. Analysis of spinal mobility and posture among dentists and dental hygienists in Slovakia. Hrvat Rev Za Rehabil Istraz. 2016;52(2):23-9.

28. Muthuraj TS, Raja J, James JR, Raj JPM, Subramnian D, Varatharajan A. Standardized photometric assessment method: A novel approach for the analysis of dental ergonomic posture. J Indian Soc Periodontol. 2020;24(3):227-32.

29. Raja RS, Gowda TM, Kumar TAB, Arya K, Mehta DS. Assessment of interns and postgraduate dental student's knowledge regarding equipment ergonomics. Indian J Dent Res. 2016;27(3):256-61.

30. Plessas A, Bernardes Delgado $M$. The role of ergonomic saddle seats and magnification loupes in the prevention of musculoskeletal disorders. A systematic review. Int J Dent Hyg. 2018;16(4):430-40.

31. Arthisri AS, Jain AR, Abraham HM, Philip JM, Venkatakrishnan CJ, Chandran CR. Dental practice-related factors associated with occupational musculoskeletal complaints among dentists in Chennai city. Drug Invent Today. 2018;10(Special Issue 3):3434-6.

32. Moodley R, Naidoo S, van Wyk J. The prevalence of occupational health- related problems in dentistry: A review of the literature. J Occup Health. 2018;60(2):111-25.

33. Gupta D, Mathur A, Patil GI, Tippanawar HK, Jain A, Jaggi N. Prevalence of musculoskeletal disorder and alternative medicine therapies among dentists of North India: A descriptive study. Pharmacogn Res. 2015;7(4):350-4.

34. Meisha DE, Alsharqawi NS, Samarah AA, Al-Ghamdi MY. Prevalence of work- related musculoskeletal disorders and ergonomic practice among dentists in Jeddah, Saudi Arabia. Clin Cosmet Investig Dent. 2019; 11:171-9. 
35. Diaz-Caballero AJ, Gómez-Palencia I-P, Díaz-Cárdenas S. Ergonomic factors that cause the presence of pain muscle in students of dentistry. Med Oral Patol Oral Cirugia Bucal. 2010;15(6): e906-11.

36. Kar G, Mullick A. Designing with users: A case study for design of dental workspace. En 2012. p. 652-5. Disponible en: https://www.scopus.com/inward/record.uri?eid=2-s2.0$84873423689 \&$ doi $=10.1177 \% 2 f 1071181312561136 \&$ partnerlD $=40 \& \mathrm{md} 5=915$ 46373d5144cb7aa3b1cadfcd05168

37. Annaç-Asildağ M, Özçirpici B. Occupational health problems and protective applications of dentists. Turk Klin J Med Sci. 2016;36(4):204-15.

38. Shadmehr A, Haddad O, Azarnia S, Sanamlo Z. Disorders of the musculoskeletal system among Tehran, Iranian dentists. J Musculoskelet Pain. 2014;22(3):256-9.

39. Taib MFM, Bahn S, Yun MH, Taib MSM. The effects of physical and psychosocial factors and ergonomic conditions on the prevalence of musculoskeletal disorders among dentists in Malaysia. Work. 2017;57(2):297- 308.

40. Lordache C, Fătu AM, Chirieac R, Ancuța C. Work-related hand pathology among dentists: A pilot study. Int J Biol Biomed Eng. 2016; 10:225-8.

41. Majeed S, Warraich HALI, Majeed F, Azeem M. Knowledge, attitude and practices about posture ergonomics among dental surgeons of Punjab Dental Hospital (PDH). Pak J Med Health Sci. 2018;12(3):977-9.

42. Mills ME, Smilyanski I, Giblin-Scanlon L, Vineyard J. What are the effects of photographic self-assessment on students' risk for musculoskeletal disorders using Rapid Upper Limb Assessment. J Dent Educ. 2020;84(7):749-54.

43. Partido BB, Henderson RP, Kennedy M. Improving the Awareness of Musculoskeletal Disorder Risks Among Dental Educators. J Dent Educ. 2020;84(1):5-12.

44. Shekhawat K, Chauhan A, Sakthidevi S, Nimbeni B, Golai S, Stephen L. Work-related musculoskeletal pain and its self-reported impact among practicing dentists in Puducherry, India. Indian J Dent Res. 2020;31(3):354-7.

45. Alghadir A, Zafar H, lqbal ZA. Work-related musculoskeletal disorders among dental professionals in Saudi Arabia. J Phys Ther Sci. 2015;27(4):1107-12. 
46. Bitencourt RS, Ricardo MI. Prevalence of discomfort/pain complaints and related factors in dental professionals of the public health service in Araucária, Paraná state, Brazil. Work. 2012;41(SUPPL.1):5715-7.

47. Bedi HS, Moon NJ, Bhatia V, Sidhu GK, Khan N. Evaluation of musculoskeletal disorders in dentists and application of DMAIC technique to improve the ergonomics at dental clinics and meta-analysis of literature. J Clin Diagn Res. 2015;9(6): ZC01-3.

48. Hodacova L, Sustova Z, Cermakova E, Kapitan M, Smejkalova J. Self- reported risk factors related to the most frequent musculoskeletal complaints among Czech dentists. Ind Health. 2015;53(1):48-55.

49. Jonker D, Rolander B, Balogh I, Sandsjö L, Ekberg K, Winkel J. Mechanical exposure among general practice dentists in Sweden and possible implications of rationalisation. Ergonomics. 2011;54(10):953-60.

50. Chamani G, Zarei MR, Momenzadeh A, Safizadeh H, Rad M, Alahyari A. Prevalence of musculoskeletal disorders among dentists in Kerman, Iran. J Musculoskelet Pain. 2012;20(3):202-7.

51. De Ruijter RAG, Stegenga B, Schaub RMH, Reneman MF, Middel B. Determinants of physical and mental health complaints in dentists: A systematic review. Community Dent Oral Epidemiol. 2015;43(1):86-96.

52. Johnson CR, Kanji Z. The impact of occupation-related musculoskeletal disorders on dental hygienists. Can J Dent Hyg. 2016;50(2):72-9.

53. Musculoskeletal disorders and dental practice: Adopted by the FDI general assembly: 26th October 2007, Dubai, UAE. J Stomatol. 2015;68(2):233-5. 